\title{
Correlación entre diagnósticos, resultados e intervenciones de enfermería en el cuidado al paciente hospitalizado por COVID-19
}

Revista Cuidarte Rev Cuid. Ene. - Abril. 2021; 12(1): e1944 doi) $h$ ttp://dx.doi.org/10.15649/cuidarte.1944

E-ISSN: 2346-3414
1 Profesora Asociada, Facultad de Enfermería, Universidad de Antioquia, Medellín, Colombia. Autor de Correspondencia: E-mail: aluz.rodriguez@udea.edu.co

2 Facultad deEnfermería, Universidad de Antioquia, Medellín, Colombia. E-mail: daniela.yampuezang@ udea.edu.co

3 Profesor Asociado, Facultad de Enfermería, Universidad de Antioquia, Medellín, Colombia. E-mail:

wilson.canon@udea.edu.co
La pandemia de COVID-19 confirmada por la Organización Mundial de la Salud (OMS) en el año 2020 es conocida como una enfermedad respiratoria infecciosa causada por un nuevo virus perteneciente a la familia coronaviridae, posee un genoma de ácido ribonucleico (ARN) de gran tamaño y simetría helicoidal, su característica definitoria son las espículas que posee en su envoltura vírica que le dan la forma de corona; así mismo, le permiten junto con las proteínas que se encuentran en la envoltura poder anclarse a los receptores de la célula'.

Se conoce que los diversos coronavirus como el Síndrome Respiratorio Agudo Grave (SARS), Síndrome Respiratorio del Medio Oriente (MERS) y actualmente el COVID-19 han sido causantes desde resfriados comunes hasta enfermedades más graves que han llevado a complicaciones y derivado en la muerte de un gran número de personas, el SARS en el 2002 causó 8300 enfermos y 785 muertes, el MERS en el 2012 reportó 1879 casos con una tasa de mortalidad de $39 \%^{2}$; la OMS ha realizado un monitoreo para reportar diariamente los casos confirmados y muertes por COVID-19 en las diferentes regiones del mundo3; esto teniendo en cuenta que la enfermedad tiene una tasa de reproducción de $\mathrm{Ro}=2.28$ originada por su rápida propagación en comparación con otros coronavirus ${ }^{4}$, que se facilita por su fácil vía de trasmisión a través de las gotas respiratorias (aerosoles) y el contacto directo e indirecto por fómites contaminados de dichos aerosoles ${ }^{5}$, unido a la falta de adherencia a las medidas de bioseguridad por parte de la población, lo cual han ocasionado un desborde en el control del virus.
Recibido: septiembre 30 de 2020

Aceptado: octubre 2 de 2020

Publicado: noviembre 13 de 2020 $\square *$ Correspondencia

Alba Luz Rodríguez-Acelas

E-mail:aluz.rodriguez@udea.edu.co 
Estos datos crecientes han desencadenado una crisis a nivel mundial que compromete diferentes ámbitos en la población; en lo que respecta a salud, si bien, la OMS divulgó algunas recomendaciones de prevención, cada país ha dispuesto sus medidas de control para evitar la rápida propagación y colapso del sistema de salud. Se conoce que algunas personas pueden cursar asintomáticas, otras con síntomas leves o moderados que requieren cuidados en casa o áreas de asistencia no críticas, pero hay un número significativo de personas que presentan un acelerado deterioro y requieren manejo en la Unidad de Cuidado Intensivo, consideradas áreas con capacidad limitada por los requerimientos de equipos y recurso humano entrenado, llevando a un alto costo en sus estancias 4 .

En este contexto, enfermería viene asumiendo un reto en los diferentes campos de acción; en el área comunitaria, busca favorecer las medidas de autocuidado como forma de empoderamiento hacia la población; sin embargo, estas dependen en gran parte de la adherencia que cada individuo tiene hacia las medidas de aislamiento social, lavado de manos, uso de tapabocas, distanciamiento social, evitando aglomeraciones, entre otros ${ }^{6}$; además, de las medidas de aislamiento específicas para la población más vulnerable y con alto riesgo de mortalidad frente a la enfermedad, como son los adultos mayores, personas con padecimientos crónicos o problemas de inmunodepresión a causa de enfermedades secundarias a tratamientos ${ }^{1}$, para las cuales, se implementó el aislamiento preventivo obligatorio, con el fin de evitar complicaciones.

En lo que concierne a las áreas hospitalarias, la dinámica de los cuidados es cambiante y eso depende de la complejidad del estado del paciente, la infección puede progresar hasta la forma más severa de la enfermedad que incluye disnea y dolor torácico, compatibles con neumonía en el 75\% de los casos $^{7}$. El periodo entre el inicio de los síntomas por COVID-19 hasta la muerte varía entre los 6 y los 41 días, con una mediana de 14 días y cambia dependiendo de la edad y del estado inmune del paciente ${ }^{8}$. Todas esas variables inclinan la balanza hacia un cuidado crítico, lleno de grandes desafíos y vulnerabilidades para los profesionales y pacientes.

En estecomplejo contexto, enfermería asume un rollleno de desafíosy centradoen la dignificación del cuidado en todas las áreas de atención; con todo, los mayores obstáculos está en el tránsito del cuidado al paciente hospitalizado por COVID-19, por un lado, está la constante necesidad del profesional en ampliar la esfera de conocimientos, ante un virus que evoluciona y transforma la mirada habitual al cuidado; por otro parte, está el paciente lleno de incertidumbres y disconfort, pero con una necesidad imperativa de un cuidado integral y coherente con esta nueva realidad, en la cual prima el salvaguardar la vida y el restablecimiento de su salud.

Toda esta situación ha develado el rol protagónico que enfermería sustenta a través de un cuidado individualizado, planificado y respaldado a través de la producción y validación de conocimientos propios de la disciplina y de la práctica profesional en todos los
Toda esta situación ha develado el rol protagónico que enfermería sustenta a través de un cuidado individualizado, planificado y respaldado a través de la producción y validación de conocimientos propios de la disciplina y de la práctica profesional en todos los ámbitos, buscando cuidados pertinentes y de calidad para los pacientes, familia, cuidadores y comunidades. 
ámbitos, buscando cuidados pertinentes y de calidad para los pacientes, familia, cuidadores y comunidades ${ }^{9}$.

En esa perspectiva, el cuidado es organizado y orientado por el Proceso de Enfermería (PE) ${ }^{10}$, el cual surgió como respuesta a la necesidad de orientar la práctica en torno al pensamiento crítico y al juicio clínico como camino al logro de los resultados esperados, con el fin que los profesionales de enfermería desarrollen una atención adecuada y de forma racional en la toma de decisiones ${ }^{11}$, el PE es estructurado por diversas rutas, algunas veces el profesional solo cuenta con la posibilidad de realizarlo mentalmente; no obstante, hoy día este proceso es apoyado en algunas instituciones por los sistemas informatizados, lo que permite realizar una articulación completa a los Sistemas de Lenguaje Estandarizado (SLE): diagnósticos enfermeros (NANDA-I) ${ }^{12}$, clasificación de resultados de enfermería (NOC) ${ }^{13}$ y clasificación de intervenciones de enfermería $(\mathrm{NIC})^{14}$, cada una de estas taxonomías participa de forma definida y organizada dentro del PE .

La integración de los SLE permite una mejor visibilidad y estandarización del cuidado, por un lado, los diagnósticos favorece la consolidación del juicio clínico, los resultados llevan a realizar una medición del impacto de la asistencia y las intervenciones se enfocan en priorizar la atención que demanda el paciente, vínculo que se evidencia en la Tabla 1, donde se relacionan los principales diagnósticos, resultados e intervenciones de enfermería en el cuidado del paciente hospitalizado por COVID-19.

Tabla 1. Diagnósticos, resultados e intervenciones de enfermería identificados en el cuidado al paciente hospitalizado por COVID-19

\begin{tabular}{|c|c|c|}
\hline Diagnósticos - NANDA - I & Resultados - NOC & Intervenciones - NIC \\
\hline \multicolumn{3}{|l|}{ Dominio 2: Nutrición } \\
\hline \multicolumn{3}{|l|}{ Clase 1: Ingestión } \\
\hline \multirow{3}{*}{$\begin{array}{l}00002 \text { - Desequilibrio nutricional: } \\
\text { ingesta inferior a las necesidades } \\
\text { corporales }\end{array}$} & 1004 - Estado nutricional & 1160 - Monitorización nutricional \\
\hline & 1014 - Apetito & 1120 - Terapia nutricional \\
\hline & 1010 - Estado de deglución & 1803 - Ayuda con el autocuidado: alimentación \\
\hline 00103 - Deterioro de la deglución & $\begin{array}{l}1008 \text { - Estado nutricional: } \\
\text { ingestión alimentaria y de líquidos }\end{array}$ & 1860 - Terapia de deglución \\
\hline \multicolumn{3}{|l|}{ Clase 4: Metabolismo } \\
\hline $\begin{array}{l}00178 \text { - Riesgo de deterioro de la } \\
\text { función hepática }\end{array}$ & 0803 - Función hepática & 2380 - Manejo de la medicación \\
\hline \multicolumn{3}{|c|}{ Dominio 3: Eliminación e intercambio } \\
\hline Clase 2: Función gastrointestinal & 1015 - Función gastrointestinal & 0460 - Manejo de la diarrea \\
\hline 00013 - Diarrea & 0501 - Eliminación intestinal & 0430 - Control intestinal \\
\hline \multicolumn{3}{|l|}{ Clase 4: Función respiratoria } \\
\hline $\begin{array}{l}00030 \text { - Deterioro del intercambio } \\
\text { de gases }\end{array}$ & $\begin{array}{l}0402 \text { - Estado respiratorio: } \\
\text { intercambio gaseoso }\end{array}$ & 3140 - Manejo de la vía aérea \\
\hline
\end{tabular}




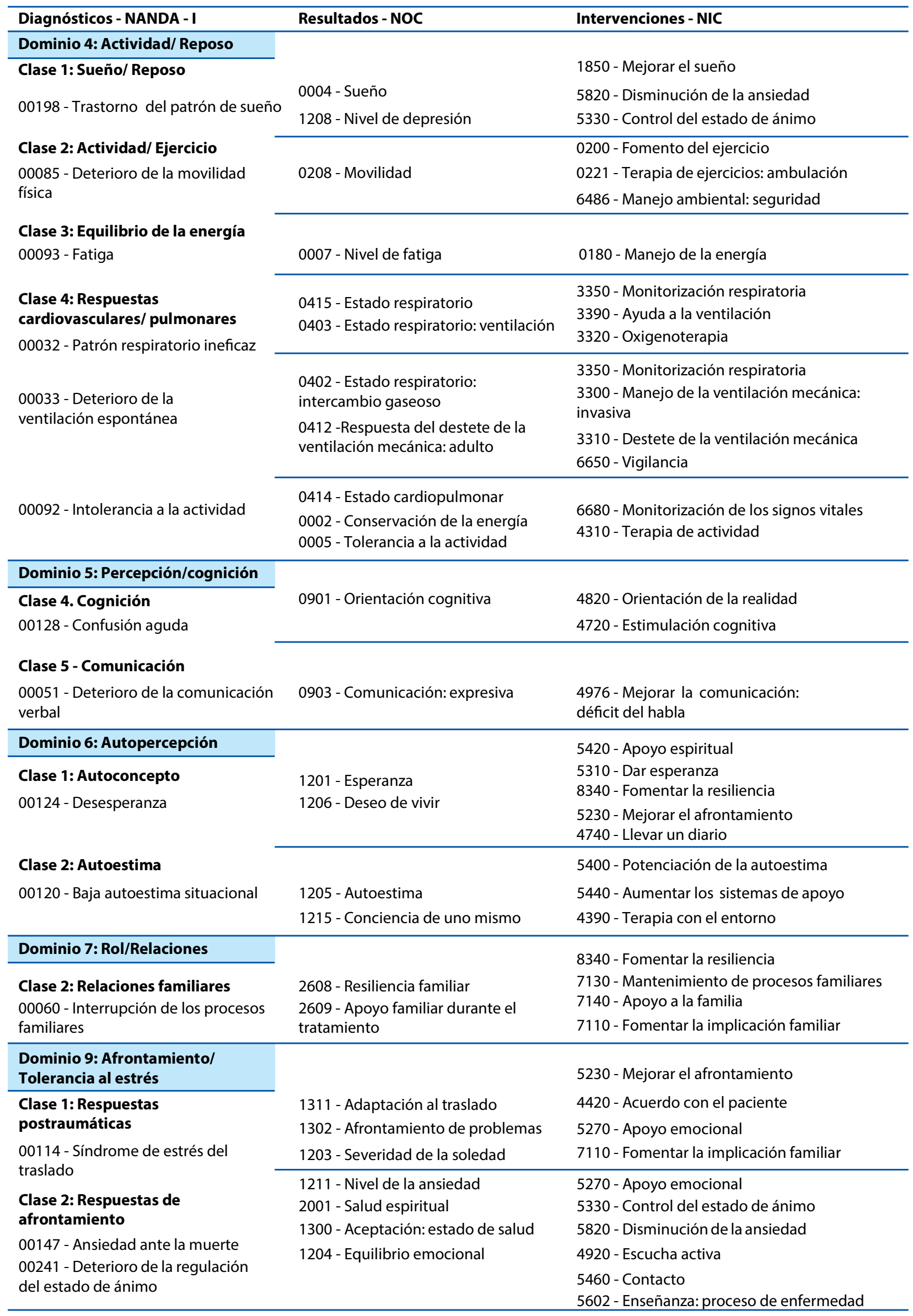




\begin{tabular}{|c|c|c|}
\hline Diagnósticos - NANDA - I & Resultados - NOC & Intervenciones - NIC \\
\hline \multicolumn{3}{|l|}{ Dominio 10: Principios vitales } \\
\hline \multirow{2}{*}{$\begin{array}{l}\text { Clase 3: Congruencia entre } \\
\text { valores/creencias/acciones }\end{array}$} & 2003 - Severidad del sufrimiento & 5420 - Apoyo espiritual \\
\hline & \multirow{2}{*}{$\begin{array}{l}2011 \text { - Estado de comodidad: } \\
\text { psicoespiritual }\end{array}$} & 5426 - Facilitar el crecimiento espiritual \\
\hline 00066 - Sufrimiento espiritual & & 5880 - Técnica de relajación \\
\hline \multirow{2}{*}{$\begin{array}{l}00242 \text {-Deterioro de la autonomía } \\
\text { para la toma de decisiones }\end{array}$} & \multirow{2}{*}{$\begin{array}{l}1606 \text { - Participación en las } \\
\text { decisiones sobre la salud } \\
0906 \text { - Toma de decisiones }\end{array}$} & 5250 - Apoyo en la toma de decisiones \\
\hline & & 7110 - Fomentar la implicación familiar \\
\hline \multicolumn{3}{|l|}{ Dominio 11: Seguridad/Protección } \\
\hline Clase 2: Lesión física & & 3590 - Vigilancia de la piel \\
\hline 00249 - Riesgo de úlcera por presión & $\begin{array}{l}1101 \text { - Integridad tisular: piel y } \\
\text { membranas mucosas }\end{array}$ & $\begin{array}{l}3540 \text { - Prevención de úlceras por presión } \\
0840 \text { - Cambio de posición }\end{array}$ \\
\hline \multirow[t]{2}{*}{00205 - Riesgo de shock } & 0416 - Perfusión tisular: celular & $\begin{array}{l}6680 \text { - Monitorización de signos vitales } \\
1910 \text { - Manejo del equilibrio ácido básico }\end{array}$ \\
\hline & 0800 - Termorregulación & 3740 - Tratamiento de la fiebre \\
\hline Clase 6: Termorregulación & 1922 - Control del riesgo: hipertermia & 3900 - Regulación de la temperatura \\
\hline 00008 - Termorregulación ineficaz & 0802 - Signos vitales & 1380 - Aplicación de calor o frío \\
\hline \multicolumn{3}{|l|}{ Dominio 12: Confort } \\
\hline Clase 1: Confort físico & & 2210 - Administración de analgésicos \\
\hline \multirow[t]{2}{*}{00132 - Dolor agudo } & 2102 - Nivel de dolor & 0840 - Cambio de posición \\
\hline & 1605 - Control del dolor & 6650 - Vigilancia \\
\hline \multirow[t]{2}{*}{00214 - Disconfort } & 2008 - Estado de comodidad & 6482 - Manejo ambiental: confort \\
\hline & & 1450 - Manejo de las náuseas \\
\hline \multirow[t]{2}{*}{00134 - Náuseas } & $\begin{array}{l}2107 \text { - Severidad de las náuseas y } \\
\text { los vómitos }\end{array}$ & 1100 - Manejo de la nutrición \\
\hline & 2301 - Respuesta a la medicación & 2300 - Administración de medicación \\
\hline
\end{tabular}

La relación entre la NANDA-I, NOC y NIC en el cuidado al paciente hospitalizado por COVID-19, es un levantamiento de datos que muestra la articulación del conocimiento disciplinar con las clasificaciones de enfermería en la práctica, visibilizando su utilidad de una forma sistemática en el cuidado direccionado a esta población, con el fin de hacer seguimiento a la evolución del cuidado de los pacientes por medio de los resultados y las intervenciones ${ }^{11}$.

Se observa en el vínculo encontrado entre las clasificaciones, que hay un gran número de dominios de la NANDA-I alterados, por lo cual se puede inferir, que de acuerdo al compromiso del paciente se pueden identificar la presencia de varios diagnósticos de enfermería, que a su vez, van conduciendo a la selección de los resultados NOC e intervenciones NIC, y que todas ellas guardan relación entre sí. Esta correspondencia entre las clasificaciones, revela la necesidad de un cuidado congruente, basado en un juicio crítico y sustentado en la producción filosófica, conceptual, teórica e investigativa propia de la profesión.

En conclusión, si bien la evidencia del cuidado conducido por el PE y soportado por las taxonomías ${ }^{12-15}$ lleva a respaldar la atención y el quehacer de los profesionales de enfermería, también se encuentra que beneficia la calidad, la optimización del tiempo, los indicadores,
Esta correspondencia entre las clasificaciones, revela la necesidad de un cuidado congruente, basado en un juicio crítico y sustentado en la producción filosófica, conceptual, teórica e investigativa propia de la profesión 
recursos y las necesidades de las personas, que finalmente, es la esencia de la profesión, donde el profesional de enfermería busca guiar el cuidado de los pacientes con COVID-19 desde un pensamiento crítico, tomando información actualizada sobre la enfermedad y contribuyendo al manejo de la pandemia, tanto del personal de la salud, como de los pacientes, desde aspectos físicos, psicológicos y sociales, que repercuten en la salud y bienestar de la población.

Conflicto de intereses: Los autores declaran no tener conflicto de intereses.

\section{Referencias}

1. Morato-Vela MC, Piédrola-Angulo G. Los coronavirus. An RANM. 2019; 136(3): 235-8. http://dx.doi.org/10.32440/ar.2019.136.03.rev01

2. Grishaw J. COVID-19. La pandemia mundial de coronavirus. Boletín: COVID-19 Ed. New York: McGraw-Hill Medical; 2020.

3. World Health Organization. WHO Coronavirus Disease (COVID-19) Dashboard. Consulta: septiembre 1, 2020. Disponible en: https://covid19.who.int/

4. Williams G, Cañon-Montañez W. COVID-19: What we've learned so far. Rev Cuid. 2020; 11(2): e1225. https://doi.org/10.15649/cuidarte.1225

5. Trilla A. One world, one health: The novel coronavirus COVID-19 epidemic. Med Clin (Barc). 2020; 154(5): 175-77. https://doi.org/10.1016/j.medcli.2020.02.002

6. Moorhead S, Macieira TGR, Lopez KD, Mantovani VM, Swanson E, Wagner C, et al. NANDA-I, NOC, and NIC Linkages to SARS-Cov-2 (Covid-19): Part 1. Community Response. Int J Nur Knowl. 2020. https://doi.org/10.1111/2047-3095.12291

7. Velavan TP, Meyer CG. The COVID-19 epidemic. Trop Med Int Health. 2020; 25(3): 278-80. https://doi.org/10.1111/tmi.13383

8. Sousa GJB, Garces TS, Cestari VRF, Florêncio RS, Moreira TMM, Pereira MLD. Mortality and survival of COVID-19. Epidemiol Infect. 2020; 148: e123. https://doi.org/10.1017/ S0950268820001405

9. Ramírez-Pereira M. El cuidado de enfermería, relevancia en el contexto de la pandemia COVID-19. Enfermería: Cuidados Humanizados. 2020; 9(1): 1-2. https://doi.org/10.22235/ech.v9i1.2184

10.0spina C, Cañon-Montañez W, Rodríguez-Acelas AL. Una mirada desde el proceso de enfermería modificado al manejo del sobrepeso y obesidad. Rev Cuid. 2020; 11(1): e1042. http://dx.doi.org/10.15649/cuidarte.1042

11.Ramírez-Elías A. Proceso de enfermería; lo que sí es y lo que no es. Enfermería Universitaria. 2016; 13(2):71-2. http://dx.doi.org/10.1016/j.reu.2016.05.001

12.Herdman T, Kamitsuru S. Diagnósticos Enfermeros: Definiciones y clasificación 2018-2020. NANDA-I. Undécima ed. España: Elsevier. 2019.

13.Moorhead S, Swanson E, Johnson M, Maas M. Clasificación de Resultados de Enfermería (NOC). Sexta ed. España: Elsevier; 2018.

14.Butcher H, Bulechek G, Dochterman J, Wagner C. Clasificación de Intervenciones de Enfermería (NIC). Séptima ed. España: Elsevier; 2018.

15. Cañon-Montañez W, Rodríguez-Acelas AL. Desarrollo de la investigación en diagnósticos de enfermería. Rev Cuid. 2010; 1(1):63-72. http://dx.doi.org/10.15649/cuidarte.v1i1.75 